\title{
Microevolution of Mitochondrial Cytochrome oxidase subunit I in Drosophila melanogaster at "Evolution Canyon", Israel
}

\author{
Nobuhiko Asada ${ }^{1}$, Hui Sun ${ }^{2}$, Kaori Hayashi ${ }^{1}$, Kohta Inomata ${ }^{1}$, Yu Harada ${ }^{1}$, Erika Sugino ${ }^{1}$, Shintaro Takasaki ${ }^{1}$ and \\ Eviatar $\mathrm{Nevo}^{3}$ \\ 1. Department of Zoology, Faculty of Science, Okayama University of Science, Kita-ku, Okayama 700-0005, Japan \\ 2. School of Life Science, Northeast Normal University, Renmin Street, Changchun 130024, China \\ 3. Institute of Evolution, Faculty of Science and Education, University of Haifa, Mount Carmel, Haifa 31905, Israel
}

\begin{abstract}
We determined the sequence of mitochondrial Cytochrome oxidase subunit I (CO1) in two Drosophila melanogaster strains originating at "Evolution Canyon”, Israel. CO1 nucleotide sequences from two iso-female strains, 2-1 and 6-1, were 1,534 and 1,543 base-pairs, respectively. In each strain, ATAA was used in initiation of translation. Exchange rates for nucleotide and amino acid sequences were larger in the 6-1 strain than in the 2-1 strain when Oregon- $\mathrm{R}$ was used as the standard. Non-synonymous exchange rate was larger than synonymous exchange rate among the three strains.
\end{abstract}

Key words: Cytochrome oxidase subunit 1, diversity, “Evolution Canyon”, drosophila melanogaster.

\section{Introduction}

For evolutionary and genetic studies, the notion of biodiversity can be understood if species diversity is considered in the context of observed patterns of genetic diversity among geographically closely isolated populations. Accumulating evidence indicates that genetic differentiation phenomena associated with ortholog evolution based on speciation and paralog evolution based on gene duplication may occur on a very small spatial scale among neighboring populations [1].

A number of studies have used mitochondrial DNA (mtDNA) sequence data to investigate genetic variation within and between species and natural populations. A crucial finding arising from these studies is that mtDNA exhibits a relatively high rate of nucleotide substitution compared to nuclear DNA;

\footnotetext{
Corresponding author: Nobuhiko Asada, Ph.D., professor, research field: population genetics. E-mail: asada@zool.ous.ac.jp.
}

consequently, mtDNA data are well suited for studies of genetic divergence among related populations and/or geographically isolated strains. The fruit fly Drosophila melanogaster (Meigen 1830) is widely distributed all over the world, except at the North and South Poles. Analysis of the rate of mtDNA exchange serves as a powerful molecular marker for the reconstruction of evolutionary lineage within species. The Cytochrome oxidase subunit I (CO1) gene is located within the mitochondria genome, indicating its ancient origins. $\mathrm{CO} 1$ is one of the most frequently utilized segments of mtDNA for genetic and evolutionary studies because 1) CO1 sequences are relatively easy to align and 2) it has been extensively characterized in numerous vertebrate taxa. Nucleotide sequences of 10 genes-COI, II, III, ATPase subunit 6 and six tRNA genes-together with two unassigned sequences have been presented; notably, the quadrupled ATAA is used in the initiation of translation [2].

The Lower Nahal Oren microsite on Mount Carmel 
in Israel, designated as "Evolution Canyon", is home to about 3,000 species [3]. The opposing slopes of this Plio-Pleistocene Canyon, which is estimated to be 3-5 million-years old, show dramatic contracts that have considerable consequences for species composition and diversity among animal and plant populations and for population genetic structure. Populations are separated by opposing slopes to cope with markedly different ecological conditions. The Nahal Oren microsite appeared to be an optimal place for studying the forces that drive evolution and adaptation [3]. In this study, mtDNA COI sequences were analyzed to assess fine-scale biodiversity among Drosophila melanogaster populations of "Evolution Canyon", Israel.

\section{Materials and Methods}

\subsection{Stations and Fly Collection}

At "Evolution Canyon", seven stations were arranged along a N-S transect line that extended across and from the top to the bottom of the south-facing slope (SFS) and the north-facing slope (NFS); the SFS housed stations 1, 2, and 3; the NFS housed stations 5, 6, and 7.

\subsection{Flies and Chemicals}

D. melanogaster were reared on a standard cornmeal-yeast medium at $25^{\circ} \mathrm{C}$.

The wild-type laboratory strain Oregon-R served as the control. The iso-female line 2-1 was established from a specimen collected on the SFS; iso-female line 6-1 was established from a specimen collected on the NFS. DNA Isolation Reagent, DNA zol, was purchased from Gibco BRL, Life Technologies, Galthersberg, MD, U. S. A.. Thermo Seaquenase Cy5.5 Dye Terminator Cycle Sequencing Kit was from Amersham Biosciences, Uppsala, Sweden.

\subsection{Extraction and Purification of $m t D N A$}

DNA sol Reagent (Genomic DNA isolation reagent, Life technologies, Buckinghampshiere, UK) was used according to manufacturer instructions with some modifications to isolate mtDNA from five live females of each strain [4]. The animals were ground with a glass motor. The crude DNA sample was purified with a spin column (Freeze ' $N$ Squeeze DNA Gel Extraction Spin Columns, Bio-Rad Laboratories Inc., Hercules, CA, USA). Concentration of DNA was measured via electrophoresis through a 1.5\% agarose gel (TaKaRa LOS, Takara, Kyoto, Japan) using a Mupid kit (Advance Co. Ltd., Tokyo, Japan) and a spectrophotometer system (U-2000 system, Hitachi, Tokyo, Japan).

\section{PCR Conditions}

MtDNA encoding CO1 was amplified by PCR using specifically designed oligonucleotide primers. To amplify the full-length of COI (1,069-2,609 bp), sense and anti-sense oligonucleotide primers were constructed based on the control sequence [2, 4]. Primer sequences used in this study were as follows:

Forward primer:

DCOF0 5' CAA TAA TCG CGA CAA' 3'

DCOF3 5' CAA ATC ATA AAGATA TTG CAA 3'

DCOF1 5' CTT TAT ATT TTA TTT TTG GGG C 3'

DCOF2 5’ CAG CGCGAG GAG GAG ATT C 3'

Reversed prime: DCOR2 5' CAT TAT TTT CTA CAA 3'

DCORS1 5' CAA CAG GAA TTT CAT TAG ATC GT 3'

DCOR1 5' TGA TAC CAA AAT ACT CCG CCA GCT GAA CAT AG 3'

DCONR1 5' GAA TTA CCA CTT TTA ACA AAT 3'

\subsection{DNA Sequence}

MtDNA sequence was obtained via the dye-terminating method [5] using a Sequanase version 20 Kit (USB). Sequence data (Fig. 1) were analyzed with GENETYX version 9 and MEGA version 6 . Genetic sequence by de Bruijin [2] was used as the 
control.

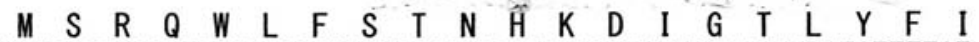

Oregon-R ATAATCGCGACAATGATTATTTTCTACAAATCATAAAGATATTGGAACTTTATATTTTAT

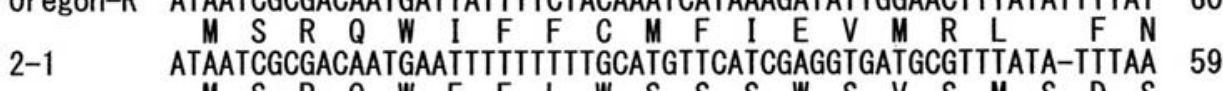

M S R Q W E E L W S S S W S V S M S D S

6-1 ATAATCGCGACAATGAGAGGAGTTGTGGAGgTCGAGATGGAGAGTCAGTATGAGAGATAG 60

$\begin{array}{ccccccccccccccccccccc}F & G & A & W & A & G & M & V & G & T & S & L & S & I & L & I & R & A & E & L\end{array}$ F G Y G G M G S L S I L I R A E L 2-1 ITTCGGAG-TTACGGTGGAATAG-TGGAA-ATCTTTAAGAATTTTAATTCGAGCTGAATT

6-1 ACAGTGTACAACGGTCTTATCGTTACCGGGCTACCGTTCCGAGCGAGTCATACAGGCAGC 120

G $\quad H \quad P \quad G \quad A$ L I G Oregon-R AGGACATCCTGGAGCATTAATTGGAGATGATCAAATTTATAATGTAATTGTAACTGCACA G H P G A L I G D D Q I Y N V I V T A H 2-1 AGGACATCCTGGAGCATTAATTGGAGATGATCAAATTTATAATGTAATTGTAACTGCACA

6-1 $\quad \begin{aligned} & \text { Y } \\ & \text { TTACTCTCCGAGAGTGACGTCAAATTTCATAATTAGCTGTATAAGCTACACAGTCACATA }\end{aligned}$

$\begin{array}{llllllllllllllllllll}A & F & I & M & I & F & F & M & V & M & P & I & M & I & G & G & F & G & N & W\end{array}$ Oregon-R TGCTTITATTATAATTTITTTTATGGTTATACCTATTATAATTGGTGGATTTGGAAATTG $\begin{array}{llllllllllllllllllll}A & F & I & M & I & F & F & M & V & M & P & I & M & I & G & G & F & G & N & W\end{array}$ 2-1 TGCTTTTATTATAATTTTTTTTATAGTTATACCTATTATAATTGGTGGATTTGGAAATTG 6-1 TTGTCATTACTCAATTTTTTTTATACTTATACGCTCATTCATAATTCCTCAATTCTGGAA 180

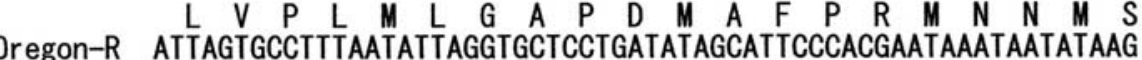
ATTAGTGCCTTTAATATTAGGTGCTCCTGATATAGCATTCCCACGAATAAATAATATAAG L V P L M L G A P D M A F P R M N N M S 2-1 ATTAGTGCCTTTAATATTAGGTGCTCCTGATATAGCATTCCCACGAATAAATAATATAAG

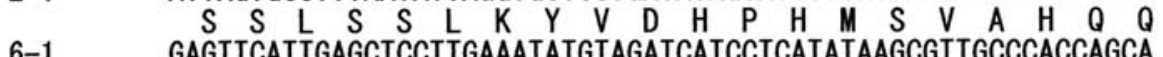
6-1 GAGTTCATTGAGCTCCTTGAATATGTAGATCATCCTCATATAAGCGTTGCCCACCAGCA

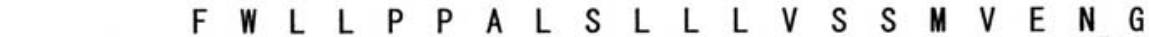
Oregon-R ATTTTGACTACTACCTCCTGCTCTTTCTTTACTATTAGTAAGTAGAATAGTTGAAAATGG

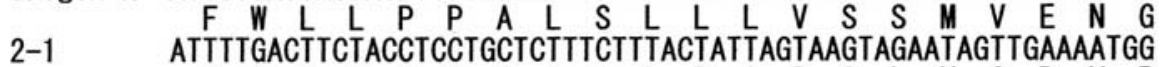
I S T K P S K A S L R S R Q L Y G D V R 6-1 AATCAGTACTAAACCTTCTAAGGCTTCATTACGTTCACGTCAGTTGTACGGGGACGTACG 360

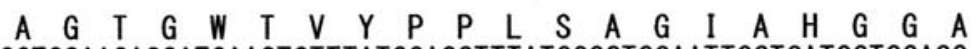
Oregon-R AGCTGGAACAGGATGAACTGTTTATCCACCTTTATCCGCTGGAATTGCTCATGGTGGAGC $A$ G T G W T V Y P P L S A G A H G G R 2-1 AGCTGGGACAGGATGAACTGTTTATCCACCTCTATCCGCTGGA-TTGCTCATGGTGGACG

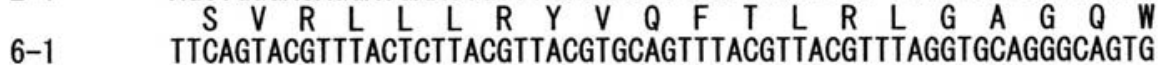

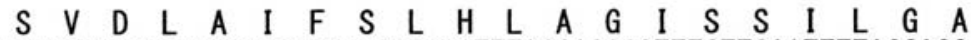
Oregon-R TTCAGTTGATTTAGCTATTTITTCTCTACATTTAGCAGGGATTTCTTCAATTTTAGGAGC $S$ V D L A I F L H L A G S S I L G A 2-1 TTCAGTTGATTTIAGCTATTTTT-CTCTACATTTAGCAGGAA-TTCTTCAATTTTAGGAGC

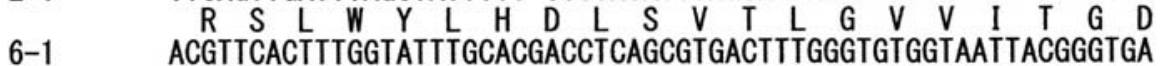
480 473 480

$\begin{array}{cccccccccccccccccccc} & V & N & F & I & T & T & V & I & N & M & R & S & T & G & I & S & L & D & R\end{array}$ V N F I T T V I D M R S T G I F I R L R 2-1 TGTAAATITTATTACAACTGTAATTGATATACGATCAACAGGAATTTTCATTCGATTACG

6-1 537 


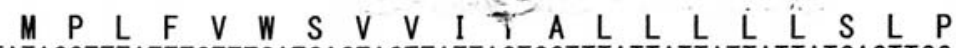

Oregon-R TATACCTTTATTTGTTTGATCAGTAGTTATTACTGCTTTATTATTATTATTATCACTTCC

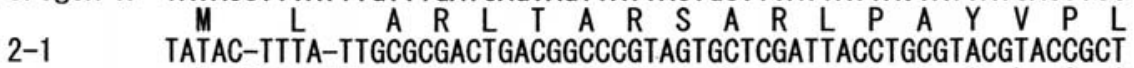

$M$ M $P$ L $F$ V

6-1 CATACCTTTATTTGTTTGACATGTTATCGTACGTTTACTGACGTTACGTCAGTTATCAGC

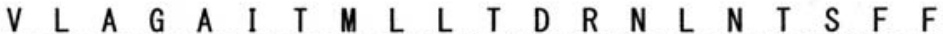

Oregon-R AGTACTAGCAGGAGCTAATTACTATATTATTACAGATCGAAATTTAAATACATCATTTTT $\begin{array}{llllllllllllllllllll}V & L & L & C & T & A & S & T & P & T & T & D & R & N & P & Y & V & A & V & V\end{array}$

2-1 AGTACTGCTATGCACCGCTAGCACCCCGACGACGGATCGAAATCCGTACGTAGCGGTCGT

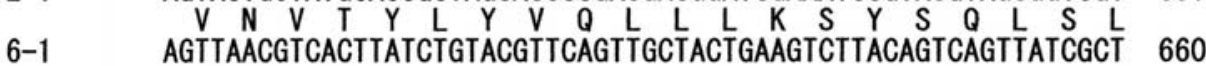

$\begin{array}{ccccccccccccccccccccc}D & P & A & G & G & G & D & P & I & L & Y & 0 & H & L & F & W & F & F & G & H\end{array}$ D $V$ V L V V C Y P V V L R H R G V D P V A 2-1 AGACGTGCTAGTCGTGTGCTACCCCGTCGTCCTGCGGCATCGAGGCGTAGACCCCGTAGC

6-1 VI T $\underset{\text { AGTAACGTGCATTCTAGACGTAACGTCGATCAACTGAGCTTTCGAGTCAATATACGATCT }}{C}$ Oregon-R CCCTGAAGTTTATATTTTAATTTTACCTGGATTTGGAATAATTTCTCATATTATTAGACA

2-1 CCCGCTGACCCCGAGACCCGTACCTGACCCCGTATTTCCCGTAGCACCCCGTATGACCCG

6-1 II V S I 771

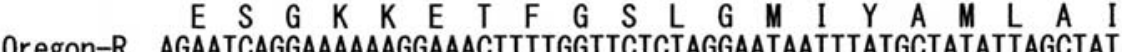

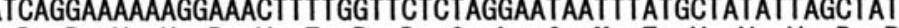

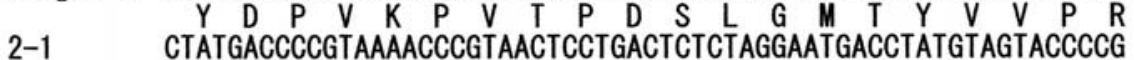

$\begin{array}{llllllllllllllllllll}D & V & Q & W & S & G & S & L & R & 0 & L & R & I & V & T & A & V & R & R & M\end{array}$

6-1 TGACGTTCAGTGATCTGGATCCCTACGTCAGCTTCGTATCGTCACTGCAGTTCGCAGAAT 837

Oregon-R TGGATTATTAGGATTTATTGTATGAGCTCATCATATATTTACCGTTGGAATAGATGTAGA 897

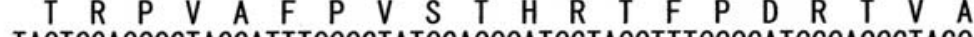

R I A R S Y P F V V P T F A V S S V L E

6-1 ACGCATTGCACGTTCGTATCCCTTCGTCGTTCCGACGTTCGCAGTCTCGTCAGTTCTCGA

Oregon-R TACTCGAGTTATITACCTCAGTACTATAATIATTA $P V B$ A $R$ M T P A T M I I A V R T R 2-1 ACCCGTACGAGCTCGTATGACCCCCGCTACTATGATTATTGCAGTACGTACCCCGCATCG

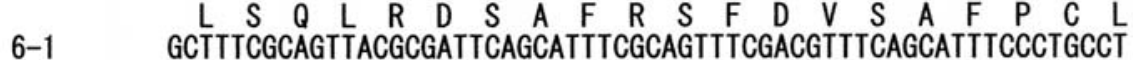
957

Oregon-R TITTAGTTGATTAGCTACTTTACATGGAACTCAACTITCTTATTCTCCAGCTATTTTATG

P A M H P M T P Y A P Y A P D A P V L T 2-1 ACCCGCTATGCACCCGATGACCCCGTATGCACCGTATGCACCCGATGCACCCGTACTGAC

6-1 $\quad P$ T T I I S M A 1017 1011 1020

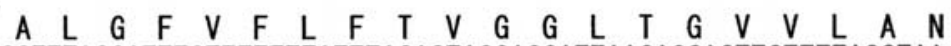
Oregon-R AGCTTTAGGATITGTTTITTTATTTACAGTAGGAGGATTAACAGGAGTTGTTTTAGCTAA R T L A P V F L T P Y V R P V D P V C P 2-1 CCGTACTTTAGCACCCGTATITTTAACCCCGTACGTACGCCCCGTAGACCCCGTATGCCC 1077

6-1 CACTTCGACCTGCACTTTCGACCCTGAATGATTTAAAGGTGTTCTAGCCGAAGTGGCATC 


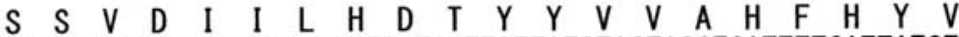
Oregon-R TTCATCAGTAGATATTATITTACATGATACTTATTATGTAGTAGCTCATTTTCATTATGT $Y$ A P R D P V L P D T D Y V C T P Y R T 2-1 GTATGCACCCCGAGACCCCGTATTACCCGATACTGACTATGTATGCACCCCGTACCGTAC 6-1 GTTCTTTAGTGGGGAGGTAACCACAGGCTGTTCGTCGGCCCGTACGTCGCACGAAAGATG 1137

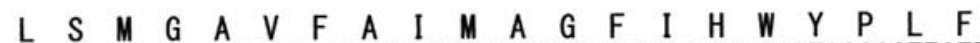
Oregon-R TTTATCTATAGGAGCTGTATTTGCTATTATAGCAGGTTTTATTCACTGATACCCCTTATT $\begin{array}{lllllllllllllllllllll}A & L & C & P & V & A & P & Y & A & R & I & A & R & M & T & R & H & R & P & V\end{array}$ 2-1 CGCTTTATGCCCCGTTGCCCCGTATGCCCCGATCGCCCGTATGACCAGTCATCGACCCGT

6-1 AGGGTGCATGTGCAAGACCCGTGAATTGTCAGGCGCCCGGTCGGTTAAGCCCAAATCTCG

Oregon-R $S T$ T $S T P$ I A P V L P R T R P V L P D 2-1 CAGCACCGCTAGTACCCCGATCGCCCCGGTATTACCCCGTACTCGCCCCGTACTGCCCGA $\begin{array}{llllllllllllllllllll}S & G & M & R & C & W & M & Q & R & W & Q & H & A & V & D & W & V & Y & D & R\end{array}$ 6-1 AAGAGGGATGCGGTGTTGGATGCAACGGTGGCAACACGCGGTAGATTGGGTGTATGATCG

$\begin{array}{llllllllllllllllllll}V & N & L & T & F & F & P & Q & H & F & L & G & L & A & G & M & P & R & R & Y\end{array}$ Oregon-R AGTTAATTTAACATTTTTTCCTCAACATTITTTAGGATTGGCTGGAATACCTCGACGTTA $R P N N S P$ I A P Y A P V L P V L P R R 2-1 TCGACCCAATTTATCGCCGATCGCCCCGTATGCCCCCGTACTGCCCGTACTGCCCCGACG $S$ S V G R L A V R Q G R R V N T V H R A 6-1 AAGAAGCGTGGGGCGTCTAGCGGTGCGGCAGGGTCGTCGAGTAAATACGGTGCATCGAGC

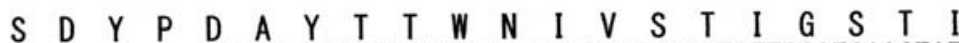
Oregon-R TTCAGATTACCCAGATGCTTACACAACATGAAATATTGTATCAACTATTGGATCAACTAT $Y R$ R Y A P Y L P L M T R H A A Y C P D 2-1 TTATCGCCGCTACGCCCCGTACTTACCCCTGATGACCCGTCATGCCGCGTATTGGCCCGA

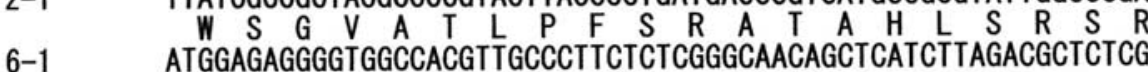

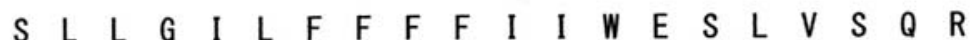
Oregon-R TTCATTATTAGGAATCTTATTCTTTTTTTTTATTATTTGAGAAAGTTTAGTATCACAACG $\begin{array}{llllllllllllll} & D & P & Y & R & G & V & L & P & V & C & T & R & L\end{array}$ 2-1 6-1 TCAGTGGGTTTCAGTCAGGATGAGAAGGTAATTCCCCATGCGCCCAGCCCAGGTGCCCC

Oregon-R ACAAGTAATTTACCCAATTCAACTAAATTCATCAATTGAATGATACCAAAATACTCCGCC P S S N V C L H S N I M M T T R H K M V 2-1 CCCGTCATCGAACGTATGCCTCCACTCAAATATTATGATGACTACACGACACAAAATGGT

Fig. 1 Sequence of the $D$. melanogaster mtDNA fragments in the 2-1 and 6-1 strains. The coding strand is shown containing the sense sequence encoding cytochrome oxidase I (COI) gene. The gene below is shown translated into the one-letter amino acid residue above. 


\section{Results and Discussion}

\subsection{Sequence of Nucleotide and Amino Acid Residues}

COI gene fragment was identified by PCR method. The COI nucleotide sequences were 1,534 bp long in the 2-1 strain and 1,543 in the 6-1 strain; each CO1 sequence included the ATAA initiation codon (Fig. 1). The numbers of deduced amino acid residues were 512, 505, and 512 in Oregon-R, strain 2-1, and strain 6-1, respectively. The 2-1 and 6-1 CO1 nucleotide sequences have been deposited in DDBJ/EMBL/Gen Bank Data Base under the accession numbers LCO25528 (2-1 strain) and LCO25529 (6-1 strain).

Cytochrome oxidase 1 (CO1) is a cytochrome $c d_{1}$-type oxidase, while the other enzymes are cytochrome $a a_{3}$-type oxidases. In general, the cytochrome $c d_{1}$-type enzyme is a nitrite reductase that also acts as a CO. An enzyme of this type could have functioned exclusively as a nitrite reductase under conditions where molecular oxygen was not available. After molecular oxygen appeared due to photosynthesis of blue-green algae, the enzyme might have acted as a CO. However, as the enzyme is extremely labile in the presence of hydrogen peroxide, CO must have become the enzyme that is more stable in the presence of peroxide [6].

\subsection{Divergence of Nucleotide and Amino Acid Residues}

The total numbers of nucleotide and amino acid residue substitutions in the COI gene were estimated using nonparametric methods. Mathematical theory can be used to study the rate of nucleotide substitution, which also serves as a basic quantity in the study of molecular evolution. Estimating the rate of nucleotide substitution was based on the number of nucleotide or amino acid. The total number of amino acid residue substitutions was calculated using the same method used for nucleotide substitutions (Fig. 1).

The rate of nucleotide and amino acid residues revealed an orthologous genetic divergence among strains; that is, exchange rates between geographically related strains, 2-1 and 6-1, were as large as 0.72 and 0.9 for nucleotide and amino acid sequences, respectively (Table 1 ).

In addition, the rate of both synonymous and non-synonymous substitutions demonstrated large variations among the three strains. Non-synonymous substitution values were approximately 0.95, suggesting that higher isolation is currently occurring among “Evolution Canyon” populations; the values for the 2-1 and 6-1 strains were each approximately 0.95 (Table 2).

\subsection{Codon Usage Variation}

Codon usage variation among the three strains was evident for amino acid residue order. Therefore, nucleotide and amino acid residues were shown the strain specific codon usage (Table 3).

Table 1 Exchange rates of nucleotide and amino acid residue substitutions.

\begin{tabular}{lll}
\hline Strain & Nucleotide & Amino acid \\
\hline Oregon-R vs 2-1 & 0.49 & 0.64 \\
Oregon-R vs 6-1 & 0.70 & 0.89 \\
2-1 vs 6-1 & 0.72 & 0.9 \\
\hline
\end{tabular}

Table 2 Exchange rates of synonymous/non and non-synonymous substitutions.

\begin{tabular}{llll}
\hline Strain & Substitution & Synonmous & Nonsynonymous \\
\hline Oregon-R vs 2-1 & 305 & 0.04 & 0.96 \\
Oregon-R vs 6-1 & 314 & 0.06 & 0.94 \\
2-1 vs 6-1 & 299 & 0.04 & 0.95 \\
\hline
\end{tabular}


Table 3 Divergence of codon usage.

\begin{tabular}{|c|c|c|c|c|}
\hline Amino acid & Codon & Oregon-R & $2-1$ & 6-1 \\
\hline \multirow{6}{*}{ Ser(S) } & TCA & 3.51 & 1.19 & 2.92 \\
\hline & TCC & 0.19 & 0.20 & 0.78 \\
\hline & TCG & 0.19 & 0.79 & 3.11 \\
\hline & TCT & 2.14 & 0.79 & 1.36 \\
\hline & AGC & 0.00 & 0.40 & 2.33 \\
\hline & AGT & 0.78 & 0.79 & 2.14 \\
\hline \multirow{6}{*}{ Arg'(R) } & AGA(-Ser & 0.97 & 0.99 & 2.72 \\
\hline & AGG(-Ser & 0.00 & 0.00 & 0.19 \\
\hline & CGA & 1.56 & 3.37 & 1.75 \\
\hline & CGC & 0.00 & 1.39 & 1.36 \\
\hline & CGG & 0.00 & 0.20 & 1.17 \\
\hline & CGT & 0.39 & 3.17 & 4.09 \\
\hline \multirow{6}{*}{ Leu(L) } & CTA & 1.36 & 1.58 & 1.75 \\
\hline & СТC & 0.00 & 0.20 & 0.97 \\
\hline & CTG & 0.00 & 2.38 & 0.58 \\
\hline & CTT & 0.78 & 0.59 & 1.36 \\
\hline & TTA & 10.33 & 4.95 & 3.31 \\
\hline & TTG & 0.19 & 0.00 & 1.75 \\
\hline \multirow{4}{*}{ Thr(T) } & ACA & 2.53 & 0.99 & 1.36 \\
\hline & ACC & 0.39 & 4.16 & 0.78 \\
\hline & ACG & 0.19 & 0.59 & 3.50 \\
\hline & ACT & 3.31 & 2.18 & 1.56 \\
\hline \multirow{4}{*}{ Pro(P) } & CCA & 1.75 & 0.59 & 0.58 \\
\hline & CCC & 0.19 & 7.33 & 1.36 \\
\hline & CCG & 0.19 & 3.96 & 0.97 \\
\hline & ССТ & 2.73 & 1.98 & 1.36 \\
\hline \multirow{4}{*}{ Ala(A) } & GCA & 1.36 & 2.38 & 2.53 \\
\hline & GCC & 0.00 & 2.18 & 1.17 \\
\hline & GCG & 0.00 & 0.79 & 0.97 \\
\hline & GCT & 5.85 & 3.37 & 0.78 \\
\hline \multirow{4}{*}{ Gly(G) } & GGA & 7.80 & 3.37 & 0.19 \\
\hline & GGC & 0.00 & 0.40 & 0.78 \\
\hline & GGG & 0.19 & 0.20 & 1.56 \\
\hline & GGT & 1.17 & 0.79 & 1.17 \\
\hline \multirow{4}{*}{$\operatorname{Val}(\mathrm{V})$} & GTA & 3.31 & 7.92 & 2.33 \\
\hline & GTC & 0.00 & 0.99 & 1.75 \\
\hline & GTG & 0.19 & 0.79 & 1.95 \\
\hline & GTT & 2.92 & 0.99 & 2.72 \\
\hline \multirow{2}{*}{ Lys(K) } & AAA & 0.97 & 0.40 & 0.78 \\
\hline & AAG & 0.19 & 0.00 & 1.56 \\
\hline \multirow{2}{*}{ Asn(N) } & AAC & 0.00 & 0.20 & 0.39 \\
\hline & AAT & 3.51 & 2.77 & 0.58 \\
\hline \multirow{2}{*}{$\mathrm{Gln}(\mathrm{Q})$} & CAA & 1.95 & 0.40 & 0.97 \\
\hline & CAG & 0.00 & 0.00 & 3.50 \\
\hline \multirow{2}{*}{$\operatorname{His}(\mathrm{H})$} & CAC & 0.39 & 0.59 & 0.97 \\
\hline & CAT & 3.12 & 1.78 & 2.14 \\
\hline \multirow{2}{*}{ Glu’(E) } & GAA & 1.75 & 0.59 & 1.56 \\
\hline & GAG & 0.00 & 0.20 & 1.95 \\
\hline
\end{tabular}


Table 3 continued

\begin{tabular}{ccccc}
\hline Amino acid & Codon & Oregon-R & $2-1$ & $6-1$ \\
\multirow{2}{*}{ Asp(D) } & GAC & 0.19 & 1.78 & 1.36 \\
& GAT & 2.73 & 2.18 & 1.75 \\
\hline \multirow{2}{*}{ Tyr(Y) } & TAC & 0.97 & 1.58 & 1.95 \\
& TAT & 2.53 & 2.38 & 1.56 \\
\hline \multirow{2}{*}{ Cys'(C) } & TGC & 0.00 & 1.58 & 1.36 \\
& TGT & 0.00 & 0.00 & 0.97 \\
\hline \multirow{2}{*}{ Phe(F) } & TTC & 0.58 & 0.79 & 1.14 \\
\hline Met(M) & TTT & 7.02 & 2.77 & 0.78 \\
\hline \multirow{2}{*}{ Ile(I) } & ATG & 0.19 & 2.57 & 0.78 \\
& ATA(-Met & 4.48 & 2.57 & 1.56 \\
\hline Trp(W) & ATC & 0.19 & 0.79 & 1.36 \\
\hline Ter & ATT & 9.55 & 3.96 & 1.75 \\
\hline TGG & 0.00 & 0.20 & 1.36 \\
\hline & TGA(-Trp & 2.92 & 0.79 & 0.19 \\
\hline & TAA & 0.19 & 0.20 & 0.00 \\
\hline
\end{tabular}

\section{Conclusions}

The genomic diversity and genome-phenome organization in D. melanogaster have been explored using molecular techniques. The main cause of genetic difference seems to be geographic isolation of the small fly, D. melanogaster. The specificity for CO1 was determined with the enzyme sequenced from $D$. melanogaster. The molecular evolution of COI gene has also been discussed from the viewpoint of its structure and function relationship. The co-origin of the 2-1 strain and the 6-1 strain is estimated to have occurred in the Lower Nahal Oren district of the "Evolution Canyon" population, and the effect of climatic naturel selection and stochasticity then drive genetic diversity between the SFS 2-1 strain and the NFS 6-1 strain.

\section{Acknowledgments}

We appreciate Satoshi Fukuyama and Ayame Nitani from Okayama University of Science for their technical assistance.

\section{References}

[1] Nevo, E. 2001. "Evolution of Genome-Phenome Diversity under Environmental Stress.” Proceedings of the National Academy of Science, U. S. A. 98: 6233-40.

[2] M. H. L. de Bruijin 1983. "Drosophila Melanogaster Mitochondrial DNA, a Novel Organization and Genetic Code.” Nature 304: 234-41.

[3] Harry, M. E., Rashkovetsky, T., Pavlicek, S., Baker, E. M., Derzhavets, P., Capy, M-L., Cariou, D., Lachaise, N., and Asada, E. Nevo 1999. "Fine-scale Biodiversity of Drosophilidae in "Evolution Canyon" at the Lower Nahal Oren Microsite, Israel.” Biologia 54: 685-705.

[4] Miyake, H., and Watada, M. 2007. "Molecular Phylogeny of the Drosophila auraria Species Complex and Allied Species of Japan Based on Nuclear and Mitochondrial DNA Sequences.” Genes and Genetic Systems 82: 77-88.

[5] Sanger, F., Nicklen, S., and Coulson, A. R. 1977. "DNA Sequencing with Chain-terninating Inhibitors.” Proceedings of the National Academy of Sciences, U. S. A. 74: 5463-7.

[6] Yamanaka, T., Fukumori, Y., Kamita, Y., and Fujii, K. 1982. A coevolutionary aspect observed between cytochrome oxidase and cytochrome $c$, 315-329, In: ed. M. Kimura, Molecular Evolution, Protein Polymorphism and the Neutral Theory, Japan Scientific Societies Press Tokyo, Springer-Verlag, Berlin, Heidelberg, New York. 